\title{
ASSESSING OUTCOMES IN ELECTRICITY AND MAGNETISM COURSES IN ENGINEERING DEGREES. STUDENTS' PERFORMANCE ANALYSED BY BEMA
}

\author{
Tania Garcia-Sanchez ${ }^{1}$, Roser Sabater I Serra ${ }^{1}$, Ana Vidaurre ${ }^{2}$, \\ José Antonio Gómez-Tejedor ${ }^{2}$, María-Antonia Serrano², José M. Meseguer-Dueñas², \\ Soledad Bernal-Perez ${ }^{1}$, Jaime Riera ${ }^{2}$, José Molina-Mateo ${ }^{2}$, \\ Vicente Donderis Quiles ${ }^{1}, \&$ M. Amparo Gámiz-González ${ }^{2}$ \\ ${ }^{I}$ Departamento de Ingeniería Eléctrica. \\ ${ }^{2}$ Departamento de Física Aplicada \\ Universitat Politècnica de València (Spain)
}

\begin{abstract}
The field of high education is a dynamic environment constantly seeking for new methodologies and tools to promote learning and increase students' performance. Learner-centered teaching methodologies such as blended learning, problem-based learning, learning-oriented assessment, flip teaching, teamwork and effective oral and written communication are new pedagogical tools used in our instruction strategy. These innovative teaching methods can be applied jointly with classical methodologies, acting in a complementary and synergistic manner. The capacity to oversee and assess the progress of student's performance is a critical issue for the academic community. One broadly used tool for measure the student's performance in introductory physics is the Brief Electricity and Magnetism Assessment (BEMA), designed specifically as a standardized instrument to evaluate students' qualitative understanding of electricity and magnetism $(\mathrm{E} \& \mathrm{M})$ key concepts.

In order to analyze the performance of E\&M students, the research-based assessment tool BEMA was used. The E\&M course analyzed is included in the Bachelor's Degree in Industrial Electronics and Automation Engineering (DIEA) at the Universitat Politècnica de València (UPV), Spain. It is a second semester traditional curriculum in which the use of traditional textbooks and screencast have been combined. BEMA pre- and post-instruction tests were carried out at the beginning and end of the course respectively. Besides, an additional test, four months after the end of the course, was also conducted in order to analyze the persistence of the learning process.

To deepen understanding of student learning in the E\&M course, correlations of pre- and post-instruction scores have been investigated, identifying systematic trends. Learning-persistence has been introduced as an additional parameter in this analysis for the DIEA curriculum. The relevant information obtained through the BEMA test will allow teachers to adapt the educational program and teaching methodologies to improve students' performance.
\end{abstract}

Keywords: Assessment, learner-centered teaching, BEMA test, innovation effectiveness.

\section{Introduction}

In the last decades, higher education is in a process of continuous evolution. Advances in Information and Communications Technology provide with new tools to support the academic needs in different teaching and learning models (Valtonen et al., 2015). Flip teaching, blended learning, teamwork, effective oral and written communication, problem solving strategies and peer evaluation are, among others, methodologies that are used in daily instruction model (Adachi, Tai, \& Dawson, 2018; Liu et al., 2016; Shih \& Tsai, 2016; Stadler, Becker, Greiff, \& Spinath, 2016). These innovative teaching methods can be applied jointly with classical methodologies, acting in a complementary and in a synergistic manner. The capacity to oversee and assess the progress of student's performance is a critical issue to the academic community. 
In order to quantify student-learning gains after the introduction of a methodological change, several standardized assessments in introductory physics (i.e., concept inventories) have been proposed. Currently, two of the most commonly used instruments to evaluate students' qualitative understanding of key concepts in electricity and magnetism (E\&M) are the brief electricity and magnetism assessment (BEMA) and the conceptual survey of electricity and magnetism (CSEM) (Ding, Chabay, Sherwood, \& Beichner, 2006; Maloney, O’Kuma, Hieggelke, \& Van Heuvelen, 2001). Both test are essentially identical in their overall performance (Eaton, Johnson, Frank, \& Willoughby, 2019). In this paper, performance of students of E\&M courses have been analyzed through the BEMA test. This test is specifically designed to assess students' knowledge of electrical and magnetic concepts before (pre-test) and after (post-test) completing electrical and magnetic courses (Kohlmyer et al., 2009).

An important novelty compared with other works is that, in addition to the pre- and post-test, an additional test was performed four months after the end of the course to analyze the persistence of the learning process. As a result, the main finding was that two thirds of the students maintain the knowledge acquired during the E\&M course after four months without receiving any additional instruction related to the subject, but a third of the students lower their grade to the levels they had before pursuing the E\&M course. The information obtained through this tool will allow adaptation of the educational program and teaching methodologies to improve students' performance.

\section{Methods}

The E\&M course analyzed is included in the Bachelor's Degree in Industrial Electronics and Automation Engineering (DIEA) at the School of Engineering Design-Universitat Politècnica de València (UPV-Spain). Lessons of the introductory E\&M course at UPV are structured through the term in two 90 minutes lesson per week, and 2 hours laboratory session every other week. The methodology used is a combination of flip-teaching (FT) and traditional methodologies, where the university's e-learning platform was intensively used.

The data collected in this study contained the results of the students' responses to the BEMA test which was delivered three times to the same group of students. The BEMA test was administrated following the usual instructions (time limit of $45 \mathrm{~min}$, the same grade for all students who completed the test, regardless of the score achieved). The BEMA pre-test (BEMA 1) was delivered to students during the first week of the course while the post-test (BEMA 2) was delivered at the end. A third test (BEMA 3) was delivered after the summer holiday at the beginning of the second year, four months after the end of the E\&M course, in order to analyze learning persistence. The number of students who have taken the tests (BEMA 1 and BEMA 2) are 115 out of 151. The number of students that carried out the third test (BEMA 3) was 83. The comparison between the second and the first test (post-test and pre-test) provides information about the effect of the course on the E\&M knowledge of the students, while the comparison with the third one, is related to the retention of E\&M concepts (persistence after a certain period of time).

To perform an in-depth analysis, the results have been divided into terciles, grouping the students from highest to lowest grades in the final mark of the E\&M course (upper, middle and lower tercile). The results have been depicted in a range from 0 to 10.

\section{Results}

The mean value of the test results, reported in Table 1, has been calculated globally for each test (BEMA 1, BEMA 2 and BEMA 3) and for each tercile. The global mean value from the first test administrated (BEMA 1), without the students having acquired any knowledge, increases from 2.49 to 4 after finishing the course (BEMA 2). This rating increase is linked with the course learning. Four months after the end of the course (BEMA 3), the global mean value decreases to 3.56, which indicates a loss of the knowledge acquired. The same trend can be observed in the results grouped by terciles, where the mean value increases from the first test to the second one and decreases for the last test. The best results are obtained at the end of the course (BEMA 2), both in the global mean and in the terciles; however, it is worth noting that the results from BEMA 3 are higher to those obtained from BEMA 1, which is related to the persistence of learning after completing the course. 
Table 1. Average of the three BEMA tests, and average of the segmentation in the terciles.

\begin{tabular}{rccc}
\hline & BEMA 1 & BEMA 2 & BEMA 3 \\
\hline Mean & 2,49 & 4,00 & 3,56 \\
Upper tercile & 3,57 & 5,52 & 5,43 \\
Middle tercile & 2,20 & 3,36 & 3,17 \\
Lower tercile & 1,63 & 3,00 & 1,94 \\
\hline
\end{tabular}

Figure 1 shows the comparison of the results from all the students classified in terciles who performed the tests. The results BEMA 1 vs. BEMA 2 (Fig. 1a) indicate how most of the students obtain a higher score after the end of the course (BEMA 2), which is indicated by their ratings located above the unit line (red line). It can also be observed that the increase in the rating is higher in the students from the upper tercile, indicating that these students obtain a higher academic performance.

Figure 1. Rating for all the students classificed in terciles (the triangle indicates the mean value of each tercile $\pm S D$ ). a) BEMA1 vs. BEMA2. b) BEMA2 vs. BEMA3.

a)

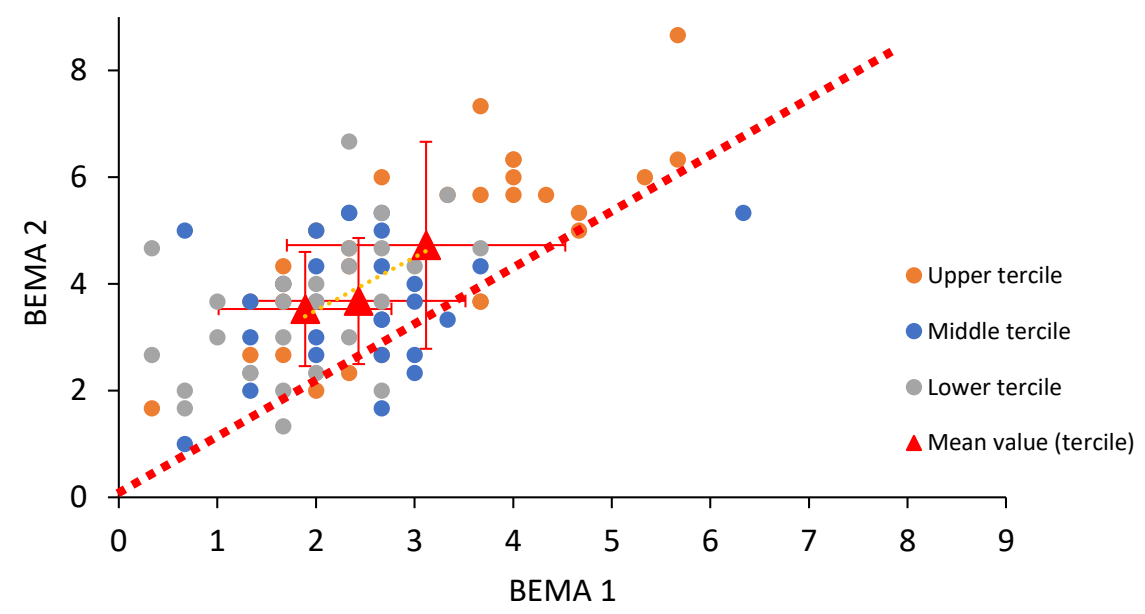

b)

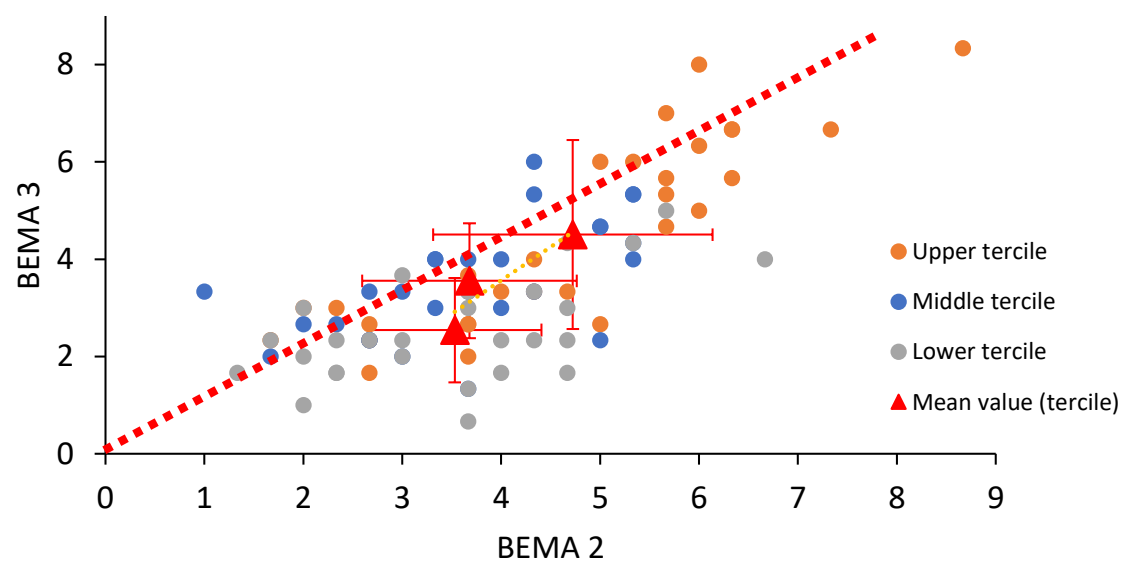

Comparing the results BEMA 2 versus BEMA 3 (Fig. 2b), it can be observed that in the upper and middle tercile, the majority of the scores are maintained close to the unit line. In particular, the mean values for both terciles are only slightly below, indicating a high persistence in the knowledge acquired. By contrast, in the lower third, a significant decrease in the mean value is observed (far away from the unit line), which indicates that the persistence drops significantly in this group of students. It can also be noted that the higher the BEMA 1 rating is, the higher rating those students obtain in the BEMA 2; likewise, the higher the BEMA 2 rating, the higher score those students obtain in the BEMA 3. These 
results suggest that the initial scores, which are related to knowledge base, lead the trend of the rest of the scores.

To analyze the learning persistence in greater detail, a Bland-Altman plot (Bland \& Altman, 1999) (Fig. 2) is used, where the semi-sum of the rating from BEMA 2 and BEMA has been represented versus the difference between them. The horizontal bars correspond to the value of the average difference (red line) and twice the standard deviation (blue lines). It can be observed how the difference increases when the semi-sum increases, indicating that students with high scores (and also medium scores) are able to keep the scores obtained four months earlier. On the other hand, students from the lower tercile are located, most of them, below the red line, pointing out the inability to maintain the previous ratings over time.

Figure 2. Bland-Altman plot from BEMA 2-BEMA 3 results.

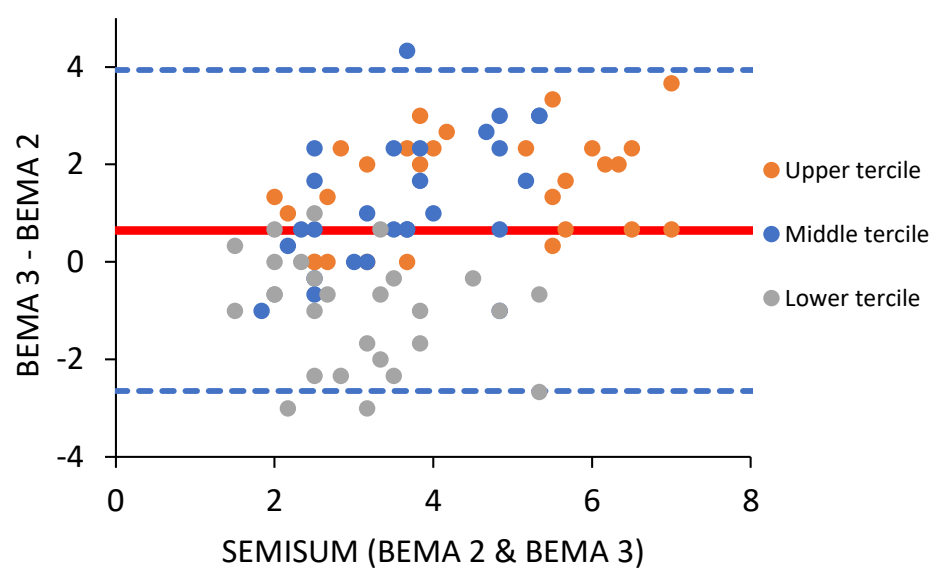

One feature worth stressing is that the rating of BEMA 3 test from the students in the lower tercile drops to values close to BEMA 1 (Table 1), indicating almost null persistence of the acquired knowledge. Conversely, students from the upper and middle tercile are able to retain the acquired knowledge. Middle tercile students presents a slight decrease in the rating (ca. 0.2 points), while for students located in the upper tercile, the difference is less than 0.1 points, which indicates that virtually all the acquired knowledge is maintained.

Figure 3. Mean rating evolution.

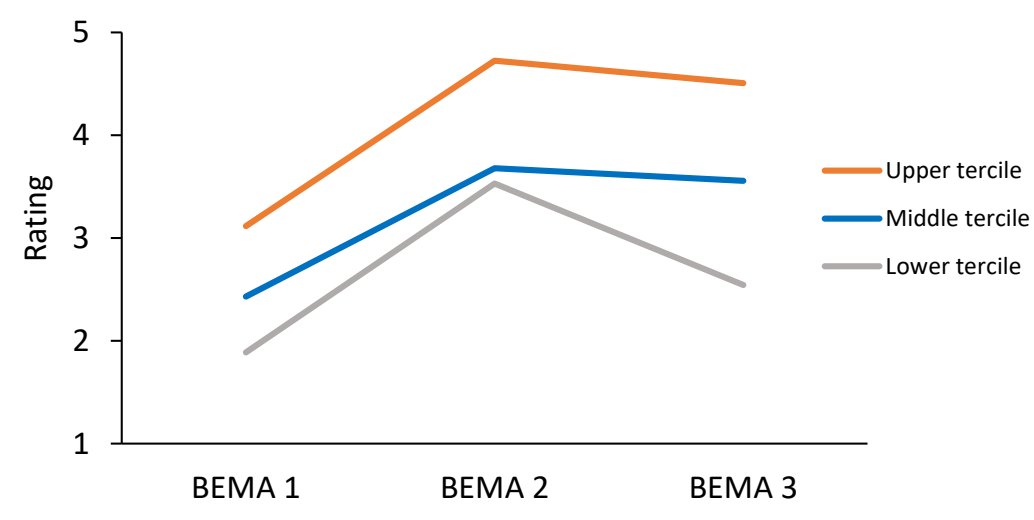

From Figure 3, where the mean rating from the different terciles has been plotted, students evolution can be clearly observed. All students increase their scores from BEMA 1 to BEMA 2, nevertheless, students whose rating grades are high or medium, maintain their grades over time while those with low ratings (lower tercile students) are not able to do so. 


\section{Conclusions}

In this paper, performance of students of E\&M courses have been analysed through the Brief electricity and magnetism assessment (BEMA), studying the correlations of pre-and post-instruction scores and identifying systematic trends both from mean results and from terciles based on their final grades in the course. The persistence of the knowledge acquired four months after the end of the course has also been included.

The results indicate a tendency in the acquisition and maintenance from what has been learned. Students who start the course with a solid knowledge base demonstrate more capacity and ability to learn throughout the course, showing higher persistence in the knowledge acquired. On the contrary, students who start the course with low knowledge base, despite increasing their knowledge, obtain lower grades because it is a superficial, not deep, learning. In addition, those students show an inability to maintain it over time.

There is a clear relationship between the types of students, the evolution of learning and the loss of it. Students with better results (higher grades) maintain their knowledge over time. On the other side, students with worse grades, with shallow learning, so volatile, lost their learning after a few months.

Effectiveness of methodological changes oriented to improve students learning have to be measured not only immediately after the course is finished, but also after some months. Persistence will also increase student's ability to incorporate new knowledge and skills.

\section{Acknowledgements}

This work has been supported by the Universitat Politècnica de València through the Project of Innovation and Educational Improvement Program (Projects PIME/2018/B26 and PIME/2018/B25 Convocatoria de Proyectos de Innovación y Convergencia).

\section{References}

Adachi, C., Tai, J. H.-M., \& Dawson, P. (2018). Academics' perceptions of the benefits and challenges of self and peer assessment in higher education. Assessment \& Evaluation in Higher Education, 43(2), 294-306. https://doi.org/10.1080/02602938.2017.1339775

Bland, J. M., \& Altman, D. G. (1999). Statistical Methods in Medical Research. Statistical Methods in Medical Research, 8(2), 161-179. https://doi.org/10.1177/096228029900800204

Ding, L., Chabay, R., Sherwood, B., \& Beichner, R. (2006). Evaluating an electricity and magnetism assessment tool: Brief electricity and magnetism assessment. Physical Review Special Topics Physics Education Research, 2(1), 010105. https://doi.org/10.1103/PhysRevSTPER.2.010105

Eaton, P., Johnson, K., Frank, B., \& Willoughby, S. (2019). Classical test theory and item response theory comparison of the brief electricity and magnetism assessment and the conceptual survey of electricity and magnetism. Physical Review Physics Education Research, 15(1), 010102. https://doi.org/10.1103/PhysRevPhysEducRes.15.010102

Kohlmyer, M. A., Caballero, M. D., Catrambone, R., Chabay, R. W., Ding, L., Haugan, M. P., ... Schatz, M. F. (2009). Tale of two curricula: The performance of 2000 students in introductory electromagnetism. Physical Review Special Topics - Physics Education Research, 5(2), 020105. https://doi.org/10.1103/PhysRevSTPER.5.020105

Liu, Q., Peng, W., Zhang, F., Hu, R., Li, Y., \& Yan, W. (2016). The Effectiveness of Blended Learning in Health Professions: Systematic Review and Meta-Analysis. Journal of Medical Internet Research, 18(1), e2. https://doi.org/10.2196/jmir.4807

Maloney, D. P., O’Kuma, T. L., Hieggelke, C. J., \& Van Heuvelen, A. (2001). Surveying students' conceptual knowledge of electricity and magnetism. American Journal of Physics, 69(S1), S12-S23. https://doi.org/10.1119/1.1371296

Shih, W.-L., \& Tsai, C.-Y. (2016). Students' perception of a flipped classroom approach to facilitating online project-based learning in marketing research courses. Australasian Journal of Educational Technology. https://doi.org/10.14742/ajet.2884

Stadler, M. J., Becker, N., Greiff, S., \& Spinath, F. M. (2016). The complex route to success: complex problem-solving skills in the prediction of university success. Higher Education Research \& Development, 35(2), 365-379. https://doi.org/10.1080/07294360.2015.1087387

Valtonen, T., Kukkonen, J., Kontkanen, S., Sormunen, K., Dillon, P., \& Sointu, E. (2015). The impact of authentic learning experiences with ICT on pre-service teachers' intentions to use ICT for teaching and learning. Computers \& Education, 81, 49-58. https://doi.org/10.1016/j.compedu.2014.09.008 